\title{
DISCRETE ASYMPTOTIC HOMOMORPHISMS IN E-THEORY AND KK-THEORY
}

\author{
KLAUS THOMSEN
}

\begin{abstract}
We obtain six-term exact sequences for E-theory and KK-theory which involve discrete asymptotic homomorphisms and generalize the extension of groups from the UCT theorem.
\end{abstract}

\section{Introduction}

In this paper we show how the homotopy classes of discrete asymptotic homomorphisms

$$
\varphi=\left(\varphi_{n}\right)_{n \in \mathrm{N}}: S A \rightarrow B \otimes \mathscr{K}
$$

can be organized to become a bivariant functor $D(A, B)$ with many of the same properties as the $E$-theory of Connes and Higson. Furthermore, we show that this bi-functor is related to $E$-theory via two six-term exact sequences of the form

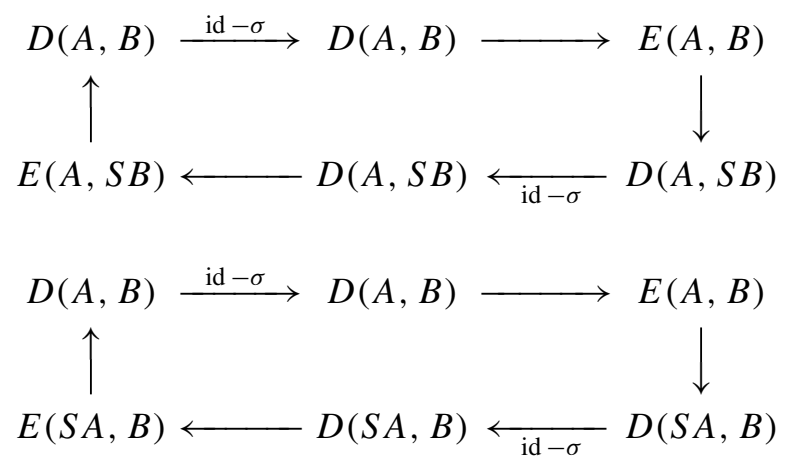

The map $\sigma$ is the automorphism induced by the shift:

$$
\sigma(\varphi)_{n}=\varphi_{n+1} .
$$

By restricting all considerations to asymptotic homomorphisms where the individual maps are completely positive linear contractions we get similar exact

Received May 19, 2000. 
sequences involving $K K$-theory, and we show how an appropriate unsplicing of one of the resulting six-term exact sequences reduces to the extension of groups from the UCT theorem in the form it has been given by Rørdam in [14] and Dadarlat and Loring in [6].

Because $E$-theory and $K K$-theory both have equivariant versions which are used in connection with the Novikov conjecture and the Baum-Connes conjecture we have taken the trouble to develop the equivariant theory and obtain the six-term exact sequences in that setting.

Although the sole purpose of this paper is to demonstrate how the theory of discrete asymptotic homomorphisms give rise to bifunctors which are intimately related to KK-theory and E-theory, we comply with the referees wish and include at the end a remark about the possibility of actually calculating these new bifunctors.

\section{Basics}

In the following $G$ will be a fixed locally compact topological group. A $C^{*}$ algebra with a pointwise normcontinuous action of $G$ by automorphisms will be called a $G$-algebra. Let $A$ and $B$ be $G$-algebras.

Definition 2.1. An asymptotic homomorphism $\varphi=\left\{\varphi_{t}\right\}_{t \in[0, \infty)}: A \rightarrow B$ is a family of functions $\varphi_{t}: A \rightarrow B$ such that

(3) $\lim _{t \rightarrow \infty}\left[\varphi_{t}(a+\lambda b)-\varphi_{t}(a)-\lambda \varphi_{t}(b)\right]=0$ for all $a, b \in A$ and all $\lambda \in \mathrm{C}$,

$$
\begin{gathered}
\lim _{t \rightarrow \infty}\left[\varphi_{t}\left(a^{*}\right)-\varphi_{t}(a)^{*}\right]=0 \text { for all } a \in A, \\
\lim _{t \rightarrow \infty}\left[\varphi_{t}(g \cdot a)-g \cdot \varphi_{t}(a)\right]=0, \quad g \in G, \quad a \in A .
\end{gathered}
$$

Note that we do not require any continuity of $\varphi_{t}$ on $A$, so in order to be able to work with asymptotic homomorphisms we need the following property. A proof can be found in [15].

Lemma 2.2. Let $\varphi=\left\{\varphi_{t}\right\}_{t \in[0, \infty)}: A \rightarrow B$ be an asymptotic homomorphism. Then

$$
\lim _{t \rightarrow \infty} \sup _{s \geq t}\left\|\varphi_{s}(y)\right\| \leq\|y\|
$$

for all $y \in A$. 
Definition 2.3. An asymptotic homomorphism $\varphi=\left\{\varphi_{t}\right\}_{t \in[0, \infty)}: A \rightarrow B$ is sequentially trivial when

$$
\lim _{n \rightarrow \infty} \varphi_{n}(a)=0, \quad a \in A .
$$

Most of the asymptotic homomorphisms $\varphi=\left\{\varphi_{t}\right\}$ we shall work with will have the following additional properties :

For all $a \in A$ and all $\epsilon>0$ there is a neighbourhood $U$ of $e$ such that

$$
\sup _{t \in[0, \infty)}\left\|g \cdot \varphi_{t}(a)-\varphi_{t}(a)\right\| \leq \epsilon, \quad g \in U .
$$

For all $a \in A$ and all $\epsilon>0$ there is a $\delta>0$ such that

$$
\sup _{t \in[0, \infty)}\left\|\varphi_{t}(a)-\varphi_{t}(b)\right\| \leq \epsilon \quad \text { when } \quad\|a-b\| \leq \delta .
$$

An asymptotic homomorphism is called equicontinuous when (6) and (7) hold.

Proposition 2.4. Let $\varphi=\left\{\varphi_{t}\right\}_{t \in[0, \infty)}: A \rightarrow B$ be a (sequentially trivial) asymptotic homomorphism. There is then a (sequentially trivial) equicontinuous asymptotic homomorphism $\varphi^{\prime}=\left\{\varphi_{t}^{\prime}\right\}_{t \in[0, \infty)}: A \rightarrow B$ such that

$$
\lim _{t \rightarrow \infty}\left[\varphi_{t}(a)-\varphi_{t}^{\prime}(a)\right]=0
$$

for all $a \in A$.

The proof of this fact is based on the following result.

THEOREM 2.5 (Brown). Let

$$
0 \longrightarrow J \stackrel{i}{\longrightarrow} E \stackrel{p}{\longrightarrow} B \longrightarrow 0
$$

be an extension of $C^{*}$-algebras. Let $\alpha: G \rightarrow$ Aut $J, \beta: G \rightarrow$ Aut $E$ and $\gamma: G \rightarrow$ Aut $B$ be homomorphisms such $G \ni g \mapsto \alpha_{g}(j)$ is continuous for all $j \in J$ and $G \ni g \mapsto \gamma_{g}(b)$ is continuous for all $b \in B$. Assume that $i$ and $p$ are $G$-equivariant, i.e. that $i \circ \alpha_{g}=\beta_{g} \circ i$ and $\gamma_{g} \circ p=p \circ \beta_{g}$ for all $g \in G$. It follows that $G \ni g \mapsto \beta_{g}(e)$ is continuous for all $e \in E$.

This result was obtained in [16] in the case where $G$ is $\sigma$-compact and the $C^{*}$-algebras separable. We will have to restrict our considerations to such cases below (in order to define the composition products), so for all our results Theorem 2.1 of [16] will suffice. However, Larry Brown has obtained the general result in [1]. In fact, Brown proves a result which is much more general than Theorem 2.5 and deals with Banach spaces. 
We can now give the

Proof of Proposition 2.4. Let $C_{b}([0, \infty), B)$ denote the $C^{*}$-algebra of bounded continuous functions $f:[0, \infty) \rightarrow B$ and consider $S B=$ $C_{0}(0, \infty) \otimes B$ as an ideal of $C_{b}([0, \infty), B)$ in the obvious way. We let $p$ : $C_{b}([0, \infty), B) \rightarrow C_{b}([0, \infty), B) / S B$ be the quotient map. The given action $\alpha: G \rightarrow$ Aut $B$ defines a representation $\beta: G \rightarrow$ Aut $C_{b}([0, \infty), B)$ in the obvious way :

$$
\beta_{g}(f)(t)=\alpha_{g}(f(t)), \quad t \in[0, \infty) .
$$

This action is generally not continuous, but its restriction to $S B$ is. $\beta$ defines a representation $\gamma$ of $G$ as automorphisms of $C_{b}([0, \infty), B) / S B$ in the obvious way, i.e. such that $\gamma_{g} \circ p=p \circ \beta_{g}$ for all $g \in G$. By Lemma 2.2 there is a map $\psi: A \rightarrow C_{b}([0, \infty), B)$ given by

$$
\psi(a)(t)=\varphi_{t}(a), \quad t \in[0, \infty) .
$$

By conditions (1)-(4) in Definition 2.1 we have that $p \circ \psi$ is a $*$-homomorphism and hence in particular continuous. By condition (5) of Definition 2.1 we have that $\psi$ is $G$-equivariant with respect to $\gamma$, and it follows that $\gamma$ restricts to a continuous representation of $G$ as automorphisms of $\psi(A)$. Then Theorem 2.5 implies that $\beta$ is continuous on $p^{-1}(\psi(A))$. By the Bartle-Graves selection theorem we can find a continuous section $s: \psi(A) \rightarrow p^{-1}(\psi(A))$ for the restriction of $p$ to $p^{-1}(\psi(A))$. Set $\varphi_{t}^{\prime}(a)=s \circ \psi(a)(t), a \in A$, $t \in[0, \infty)$. Then $\varphi^{\prime}$ is an equicontinuous asymptotic homomorphism such that $\lim _{t \rightarrow \infty}\left\|\varphi_{t}(a)-\varphi_{t}^{\prime}(a)\right\|=0$ for all $a \in A$. In particular, $\varphi^{\prime}$ is sequentially trivial when $\varphi$ is.

When $X$ is a locally compact Hausdorff space, we consider $C_{0}(X) \otimes B$ as a $G$-algebra with the action

$$
(g \cdot f)(x)=g \cdot f(x), \quad x \in X, \quad f \in C_{0}(X) \otimes B .
$$

The equivariant homomorphism $C_{0}(X) \otimes B \rightarrow B$ given by evaluation at $x \in X$ will be denoted by $\pi_{x}$. The special cases $X=(0, \infty)$ and $X=[0,1]$ will be used so often that a special notation is convenient. We shall denote the $C^{*}$ algebras $C_{0}(0, \infty) \simeq C_{0}(0,1)$ and $C[0,1]$ by $S$ and $I$, respectively, and set $S B=C_{0}(0, \infty) \otimes B, I B=C[0,1] \otimes B$.

Two (sequentially trivial) asymptotic homomorphisms $\varphi, \psi: A \rightarrow B$ are homotopic when there is a (sequentially trivial) asymptotic homomorphism $\boldsymbol{\Phi}=\left\{\boldsymbol{\Phi}_{t}\right\}: A \rightarrow C[0,1] \otimes B$ such that $\pi_{0} \circ \boldsymbol{\Phi}_{t}=\varphi_{t}, \pi_{1} \circ \boldsymbol{\Phi}_{t}=\psi_{t}$ for all $t \in[0, \infty)$. This is an equivalence relation, and we denote the set of homotopy classes of asymptotic homomorphisms by $[[A, B]]$, and the set of homotopy classes of sequentially trivial asymptotic homomorphisms by $[[A, B]]_{0}$. 
When dealing with asymptotic homomorphisms which are equicontinuous it is natural to impose the condition of equicontinuity on the homotopies also. Thus two equicontinuous (sequentially trivial) asymptotic homomorphisms $\varphi, \psi: A \rightarrow B$ are homotopic when there is a (sequentially trivial) equicontinuous asymptotic homomorphism $\boldsymbol{\Phi}=\left\{\boldsymbol{\Phi}_{t}\right\}: A \rightarrow C[0,1] \otimes B$ such that $\pi_{0} \circ \boldsymbol{\Phi}_{t}=\varphi_{t}, \pi_{1} \circ \boldsymbol{\Phi}_{t}=\psi_{t}$ for all $t \in[0, \infty)$. Let us (temporarily) introduce the notation $[[A, B]]^{u}$ and $[[A, B]]_{0}^{u}$ for the set of homotopy classes of equicontinuous asymptotic homomorphisms, and sequentially trivial equicontinuous asymptotic homomorphisms, respectively.

LemMa 2.6. The natural maps $[[A, B]]^{u} \rightarrow[[A, B]]$ and $[[A, B]]_{0}^{u} \rightarrow$ $[[A, B]]_{0}$ are bijections.

Proof. This follows readily from Proposition 2.4

Having proved this lemma we will identify $[[A, B]]^{u}$ with $[[A, B]]$, and $[[A, B]]_{0}^{u}$ with $[[A, B]]_{0}$.

\section{Products and pairings}

We are now going to define the composition product of (sequentially trivial) asymptotic homomorphisms, and a pairing between asymptotic homomorphisms and sequentially trivial asymptotic homomorphisms. For this purpose we need to restrict our considerations to separable $G$-algebras and need to assume that $G$ is $\sigma$-compact. These restrictions will be in force in the rest of the paper.

The constructions in this section are all essentially identical to the corresponding constructions in [3] and [8]. The only difference is that we have to take additional care to handle sequentially trivial asymptotic homomorphisms.

DEFINITION 3.1. A parametrization (of $[0, \infty)$ ) is a continuous non-decreasing function $r:[0, \infty) \rightarrow[0, \infty)$ such that $\lim _{t \rightarrow \infty} r(t)=\infty$.

Note that when $\varphi=\left\{\varphi_{t}\right\}: A \rightarrow B$ is an equicontinuous asymptotic homomorphism and $r:[0, \infty) \rightarrow[0, \infty)$ is a parametrization, $\varphi_{r(.)}$ is an equicontinuous asymptotic homomorphism which is homotopic to $\varphi$. But of course not necessarily sequentially trivial even when $\varphi$ is. This is the main reason that we have to take additional care in the construction of the composition product for sequentially trivial asymptotic homomorphisms.

Definition 3.2. Let $A, B$ and $C$ be $G$-algebras, and let $\varphi=\left\{\varphi_{t}\right\}_{t \in[0, \infty)}$ : $A \rightarrow B$ and $\psi=\left\{\psi_{t}\right\}_{t \in[0, \infty)}: B \rightarrow C$ be equicontinuous asymptotic homomorphisms. A composition pair for $\psi$ and $\varphi$ is a dense subset $X \subseteq A$ which is the union of a sequence of compact subsets containing 0 and a parametrization 
$r:[0, \infty) \rightarrow[0, \infty)$ such that

$$
\begin{gathered}
\lim _{t \rightarrow \infty} \sup _{s \geq r(t)}\left\|\psi_{s} \circ \varphi_{t}(a b)-\psi_{s} \circ \varphi_{t}(a) \psi_{s} \circ \varphi_{t}(b)\right\|=0, \\
\lim _{t \rightarrow \infty} \sup _{s \geq r(t)}\left\|\psi_{s} \circ \varphi_{t}(a+\lambda b)-\psi_{s} \circ \varphi_{t}(a)-\lambda \psi_{s} \circ \varphi_{t}(b)\right\|=0, \\
\lim _{t \rightarrow \infty} \sup _{s \geq r(t)}\left\|\psi_{s} \circ \varphi_{t}\left(a^{*}\right)-\psi_{s} \circ \varphi_{t}(a)^{*}\right\|=0,
\end{gathered}
$$

for all $a, b \in X, \lambda \in \mathrm{C}$, and for every compact subset $K \subseteq G$, every pair $a, b \in X$ and every $\epsilon>0$ there is a $t_{0} \in[0, \infty)$ such that

$$
\sup _{s \geq r(t)}\left\|g \cdot \psi_{s} \circ \varphi_{t}(a)-\psi_{s} \circ \varphi_{t}(h \cdot b)\right\| \leq\|g \cdot a-h \cdot b\|+\epsilon,
$$

for all $g, h \in K$ when $t \geq t_{0}$.

Note that if $(X, r)$ is a composition pair for $\varphi$ and $\psi$, then so is $\left(X, r^{\prime}\right)$ for any parametrization $r^{\prime}$ such that $r^{\prime} \geq r$.

Proposition 3.3. Let $A, B$ and $C$ be G-algebras. Let $\varphi=\left\{\varphi_{t}\right\}_{t \in[0, \infty)}$ : $A \rightarrow B$ and $\psi=\left\{\psi_{t}\right\}_{t \in[0, \infty)}: B \rightarrow C$ be equicontinuous asymptotic homomorphisms, and let $X \subseteq A$ be a dense subset which is the union of a countable family of compact sets containing 0 . There is then a parametrization $r:[0, \infty) \rightarrow[0, \infty)$ such that $(X, r)$ is a composition pair for $\psi$ and $\varphi$.

PRoOF. This follows straightforwardly by using the separability of the $C^{*}$ algebras, the $\sigma$-compactness of the group and the equicontinuity of the asymptotic homomorphisms.

Theorem 3.4 (Connes and Higson, [3]). There is a map

$$
[[A, B]] \times[[B, C]] \ni(x, y) \mapsto y \bullet x \in[[A, C]]
$$

with the following properties :

(a) (Definition): When $\varphi: A \rightarrow B$ and $\psi: B \rightarrow C$ are equicontinuous asymptotic homomorphisms, and $(X, r)$ is a composition pair for $\psi$ and $\varphi$, then

$$
[\psi] \bullet[\varphi]=[\lambda]
$$

where $\lambda: A \rightarrow C$ is any equicontinuous asymptotic homomorphism such that

$$
\lim _{t \rightarrow \infty}\left[\lambda_{t}(x)-\psi_{s(t)} \circ \varphi_{t}(x)\right]=0, \quad x \in X,
$$

for some parametrization $s \geq r$. 
(b) (Associativity):

$$
z \bullet(y \bullet x)=(z \bullet y) \bullet x,
$$

$x \in[[A, B]], y \in[[B, C]], z \in[[C, D]]$.

Proof. The proof of this is basically the same as in the case dealt with by Connes and Higson in [3]. Since all the details can be read out of the following proofs just by ignoring all references to sequential triviality we shall omit them here.

Lemma 3.5. Let $\varphi: A \rightarrow B, \psi: B \rightarrow C$ be equicontinuous asymptotic homomorphisms. Assume that $\varphi$ is sequentially trivial. Let $(X, r)$ be a composition pair for $\psi$ and $\varphi$.

(a) For any parametrization $s \geq r$ there is a sequentially trivial equicontinuous asymptotic homomorphism $\lambda^{s}: A \rightarrow C$ such that

$$
\lim _{t \rightarrow \infty} \lambda_{t}^{s}(x)-\psi_{s(t)} \circ \varphi_{t}(x)=0, \quad x \in X .
$$

$\lambda^{s}$ is unique up to asymptotic equality.

(b) When $s_{1}, s_{2}$ are parametrizations such that $s_{i} \geq r, i=1,2$, the two sequentially trivial asymptotic homomorphisms $\lambda^{s_{1}}$ and $\lambda^{s_{2}}$ are homotopic.

Proof. (a): Since $0 \in X$, it follows from conditions (9) and (11) of Definition 3.2 that

$$
\limsup _{t \rightarrow \infty}\left\|\psi_{s(t)} \circ \varphi_{t}(x)\right\| \leq\|x\|, \quad x \in X .
$$

In the notation from the proof of Proposition 2.4 this shows that every element $x \in X$ defines an element $F_{x} \in C_{b}([0, \infty), C)$ such that $F_{x}(t)=\psi_{s(t)} \circ$ $\varphi_{t}(x), t \in[0, \infty)$. In fact $F_{x} \in C_{b}^{G}(C)$, where

$$
C_{b}^{G}(C)=\left\{f \in C_{b}([0, \infty), C): g \mapsto g \cdot f \text { is continuous }\right\},
$$

because of condition (11) of Definition 3.2. Let $q: C_{b}^{G}(C) \rightarrow C_{b}^{G}(C) / S C$ be the quotient map. Since $\left\|q\left(F_{x}\right)-q\left(F_{y}\right)\right\| \leq\|x-y\|$ for all $x, y \in X$ by condition (11) of Definition 3.2, the map $x \mapsto q\left(F_{x}\right)$ extends by continuity to a map $\Lambda: A \rightarrow C_{b}^{G}(C) / S C$, and conditions (8)-(10) of Definition 3.2 ensure that $\Lambda$ is a $*$-homomorphism, and $\Lambda$ is $G$-equivariant by condition (11). By the Bartle-Graves selection theorem there is a continuous right inverse $s$ for $q$ and we set $\lambda_{t}^{s}(a)=s \circ \Lambda(a)(t), t \in[0, \infty)$. It is clear that this proves the existence of an equicontinuous asymptotic homomorphism $\lambda^{s}: A \rightarrow C$ such that

$$
\lim _{t \rightarrow \infty}\left[\lambda_{t}^{s}(x)-\psi_{s(t)} \circ \varphi_{t}(x)\right]=0, \quad x \in X .
$$


Since $\psi$ is equicontinuous and $\varphi$ sequentially trivial, it follows that $\lim _{n \rightarrow \infty} \lambda_{n}^{s}(a)$ $=0$ for all $a \in X$. By equicontinuity of $\lambda^{s}$ this must then hold for all $a \in A$, i.e. $\lambda^{s}$ is sequentially trivial. The uniqueness up to asymptotic equality follows from equicontinuity and the density of $X$.

(b) It suffices to show that $\lambda^{s}$ is homotopic to $\lambda^{r}$ for every parametrization $s \geq r$. To this end note that we may define, for each $t \in[0, \infty)$, a map $\boldsymbol{\Psi}_{t}: X \rightarrow C[0,1] \otimes C$ by

$$
\boldsymbol{\Psi}_{t}(x)(\alpha)=\psi_{\alpha s(t)+(1-\alpha) r(t)} \circ \varphi_{t}(x), \quad \alpha \in[0,1] .
$$

Since $\alpha s(t)+(1-\alpha) r(t) \geq r(t)$ for all $\alpha, t$, it follows from the conditions on $(X, r)$, that $\lim _{t \rightarrow \infty}\left\|\boldsymbol{\Psi}_{t}(a b)-\boldsymbol{\Psi}_{t}(a) \boldsymbol{\Psi}_{t}(b)\right\|=0$ for all $a, b \in X$, $\lim _{t \rightarrow \infty}\left\|\boldsymbol{\Psi}_{t}(a+\lambda b)-\boldsymbol{\Psi}_{t}(a)-\lambda \boldsymbol{\Psi}_{t}(b)\right\|=0$ for all $a, b \in X$ and all $\lambda \in \mathrm{C}$, $\lim _{t \rightarrow \infty}\left\|\boldsymbol{\Psi}_{t}\left(a^{*}\right)-\boldsymbol{\Psi}_{t}(a)^{*}\right\|=0$ for all $a \in X$, and for every compact subset $K \subseteq G$, every $a, b \in X$ and every $\epsilon>0$ there is a $t_{0} \in[0, \infty)$ such that

$$
\sup _{s \geq t}\left\|\boldsymbol{\Psi}_{s}(g \cdot a)-h \cdot \boldsymbol{\Psi}_{s}(b)\right\| \leq\|g \cdot a-h \cdot b\|+\epsilon
$$

for all $g, h \in K$ when $t \geq t_{0}$. Hence, as in the first part of the proof, we obtain an equicontinuous asymptotic homomorphism $\boldsymbol{\Phi}: A \rightarrow C[0,1] \otimes C$ such that

$$
\lim _{t \rightarrow \infty} \sup _{\alpha \in[0,1]}\left\|\boldsymbol{\Phi}_{t}(x)(\alpha)-\psi_{\alpha s(t)+(1-\alpha) r(t)} \circ \varphi_{t}(x)\right\|=0,
$$

for all $x \in X$. Since $\varphi$ is sequentially trivial and $\psi$ equicontinuous we have automatically that $\boldsymbol{\Phi}$ is sequentially trivial. Thus $\boldsymbol{\Phi}$ defines a homotopy between a sequentially trivial asymptotic homomorphism which is asymptotically equal to $\lambda^{s}$ and one which is asymptotically equal to $\lambda^{r}$. It follows that $\lambda^{r}$ and $\lambda^{s}$ are homotopic as sequentially trivial asymptotic homomorphisms.

THEOREM 3.6. There is a map

$$
[[A, B]]_{0} \times[[B, C]] \ni(x, y) \mapsto y \bullet x \in[[A, C]]_{0}
$$

with the following properties :

(a) (Definition): When $\varphi: A \rightarrow B$ and $\psi: B \rightarrow C$ are equicontinuous asymptotic homomorphisms, $\varphi$ is sequentially trivial and $(X, r)$ is a composition pair for $\psi$ and $\varphi$, then

$$
[\psi] \bullet[\varphi]=[\lambda]
$$

where $\lambda: A \rightarrow C$ is any equicontinuous asymptotic homomorphism such that

$$
\lim _{t \rightarrow \infty}\left[\lambda_{t}(x)-\psi_{s(t)} \circ \varphi_{t}(x)\right]=0, \quad x \in X,
$$


for some parametrization $s \geq r$.

(b) (Associativity):

$$
z \bullet(y \bullet x)=(z \bullet y) \bullet x,
$$

in $[[A, D]]_{0}$, when $x \in[[A, B]]_{0}, y \in[[B, C]], z \in[[C, D]]$.

Proof. (a) Let $\varphi^{\prime}: A \rightarrow B, \psi^{\prime}: B \rightarrow C$ be equicontinuous asymptotic homomorphisms such that $[\varphi]=\left[\varphi^{\prime}\right]$ in $[[A, B]]_{0}$ and $[\psi]=\left[\psi^{\prime}\right]$ in $[[B, C]]$. Let $\left(X_{1}, r_{1}\right)$ be a composition pair for $\psi$ and $\varphi$, and $\left(X_{2}, r_{2}\right)$ a composition pair for $\psi^{\prime}$ and $\varphi^{\prime}$. Let $s_{1} \geq r_{1}, s_{2} \geq r_{2}$ be parametrizations. Assume that $\lambda^{1}, \lambda^{2}$ : $A \rightarrow C$ are equicontinuous sequentially trivial asymptotic homomorphisms such that

$$
\lim _{t \rightarrow \infty}\left[\lambda_{t}^{1}(x)-\psi_{s_{1}(t)} \circ \varphi_{t}(x)\right]=0, \quad x \in X_{1},
$$

and

$$
\lim _{t \rightarrow \infty}\left[\lambda_{t}^{2}(x)-\psi_{s_{2}(t)}^{\prime} \circ \varphi_{t}^{\prime}(x)\right]=0, \quad x \in X_{2} .
$$

We must show that $\lambda^{1}$ and $\lambda^{2}$ are homotopic. Let $\boldsymbol{\Phi}: A \rightarrow C[0,1] \otimes B$ be a sequentially trivial equicontinuous asymptotic homomorphism such that $\pi_{0} \circ \boldsymbol{\Phi}=\varphi$ and $\pi_{1} \circ \boldsymbol{\Phi}=\varphi^{\prime}$. Let $\boldsymbol{\Psi}: C[0,1] \otimes B \rightarrow C[0,1] \otimes C$ be the equicontinuous asymptotic homomorphism given by $\boldsymbol{\Psi}_{t}(f)(\alpha)=\psi_{t}(f(\alpha))$, $\alpha \in[0,1], f \in C[0,1] \otimes B$. Let $\boldsymbol{\Psi}^{1}: B \rightarrow C[0,1] \otimes C$ be an equicontinuous asymptotic homomorphism with $\pi_{0} \circ \boldsymbol{\Psi}^{1}=\psi$ and $\pi_{1} \circ \boldsymbol{\Psi}^{1}=\psi^{\prime}$. Set $Y=$ $X_{1} \cup X_{2}$. By Proposition 3.3 there is a composition pair $(Y, v)$ for $\boldsymbol{\Psi}$ and $\boldsymbol{\Phi}$ and a composition pair $\left(Y, v_{1}\right)$ for $\boldsymbol{\Psi}^{1}$ and $\varphi^{\prime}$ such that $v \geq s_{1}$ and $v_{1} \geq s_{2}$. By Lemma 3.5, applied to $\Phi$ and $\boldsymbol{\Psi}$, this gives us, for every parametrization $w \geq v \vee v_{1}$, an equicontinuous sequentially trivial asymptotic homomorphism, $\lambda^{3}: A \rightarrow C$, which is homotopic to $\lambda^{1}$ and satisfies that

$$
\lim _{t \rightarrow \infty}\left[\lambda_{t}^{3}(x)-\psi_{w(t)} \circ \varphi_{t}^{\prime}(x)\right]=0, \quad x \in Y .
$$

Similarly, by applying Lemma 3.5 to $\Psi^{1}$ and $\varphi^{\prime}$, we get an equicontinuous sequentially trivial asymptotic homomorphism $\lambda^{4}: A \rightarrow C$ which is homotopic to $\lambda^{2}$ and satisfies that

$$
\lim _{t \rightarrow \infty}\left[\lambda_{t}^{4}(x)-\psi_{w(t)} \circ \varphi_{t}^{\prime}(x)\right]=0, \quad x \in Y .
$$

Since $Y$ is dense in $A, \lambda^{3}$ and $\lambda^{4}$ are asymptotically equal and hence homotopic.

(b) Let $\varphi: A \rightarrow B, \psi: B \rightarrow C, \lambda: C \rightarrow D$ be equicontinuous asymptotic homomorphisms, with $\varphi$ sequentially trivial, such that $[\lambda]=z,[\psi]=y$, $[\varphi]=x$. Let $(X, r)$ be a composition pair for $\psi$ and $\varphi$, and $\mu: A \rightarrow C$ an equicontinuous sequentially trivial asymptotic homomorphism such that 
$\lim _{t \rightarrow \infty}\left\|\mu_{t}(x)-\psi_{r(t)} \circ \varphi_{t}(x)\right\|=0, x \in X$. Let $(X, w)$ be a composition pair for $\lambda$ and $\mu$ which satisfies several additional properties which we now describe. By choosing $w$ to be sufficiently rapidly increasing we can ensure that

$$
\begin{aligned}
& \lim _{t \rightarrow \infty} \sup _{s \geq t} \| \lambda_{w(s)} \circ \psi_{r(s)} \circ \varphi_{t}(a b) \\
& \quad-\lambda_{w(s)} \circ \psi_{r(s)} \circ \varphi_{t}(a) \lambda_{w(s)} \circ \psi_{r(s)} \circ \varphi_{t}(b) \|=0, \\
& \lim \sup _{t \rightarrow \infty} \| \lambda_{w(s)} \circ \psi_{r(s)} \circ \varphi_{t}(a+z b) \\
& \quad-\lambda_{w(s)} \circ \psi_{r(s)} \circ \varphi_{t}(a)-z \lambda_{w(s)} \circ \psi_{r(s)} \circ \varphi_{t}(b) \|=0, \\
& \quad \lim \sup _{t \rightarrow \infty}\left\|\lambda_{w(s)} \circ \psi_{r(s)} \circ \varphi_{t}\left(a^{*}\right)-\lambda_{w(s)} \circ \psi_{r(s)} \circ \varphi_{t}(a)^{*}\right\|=0
\end{aligned}
$$

for all $a, b \in X, z \in \mathrm{C}$, and for every compact subset $C \subseteq G$, any pair $a, b \in X$ and any $\epsilon>0$ there is a $t_{0} \in[0, \infty)$ such that

$$
\begin{aligned}
\sup _{s \geq t}\left\|\lambda_{w(s)} \circ \psi_{r(s)} \circ \varphi_{t}(g \cdot a)-h \cdot \lambda_{w(s)} \circ \psi_{r(s)} \circ \varphi_{t}(b)\right\| \\
\leq\|g \cdot a-h \cdot b\|+\epsilon
\end{aligned}
$$

for all $g, h \in C$ when $t \geq t_{0}$. Let us show how to meet the last condition. Write $G=\bigcup_{n} G_{n}$ where each $G_{n}$ is open with $\overline{G_{n}}$ compact, and $X=\bigcup_{n} K_{n}$ where each $K_{n}$ is a compact subset of $A$. Both sequences are chosen so that they are increasing. For each $n$ there is a $t_{n}$ such that

$$
\sup _{s \geq t}\left\|\psi_{r(s)} \circ \varphi_{t}(g \cdot a)-h \cdot \psi_{r(s)} \circ \varphi_{t}(b)\right\| \leq\|g \cdot a-h \cdot b\|+\frac{1}{n}
$$

for all $g, h \in G_{n}, a, b \in K_{n}, t \geq t_{n}$. This follows from condition (11) of Definition 3.2. We may assume that $t_{n}<t_{n+1}$ for all $n$ and that $\lim _{n \rightarrow \infty} t_{n}=\infty$. By equicontinuity and compactness we can find $s_{n} \in[0, \infty)$ such that

$$
\sup _{y \geq s_{n}}\left\|\lambda_{y}\left(g \cdot \psi_{r(s)} \circ \varphi_{t}(a)\right)-g \cdot \lambda_{y} \circ \psi_{r(s)} \circ \varphi_{t}(a)\right\| \leq \frac{1}{n},
$$

and

$$
\begin{aligned}
\sup _{y \geq s_{n}}\left\|\lambda_{y} \circ \psi_{r(s)} \circ \varphi_{t}(g \cdot a)-\lambda_{y}\left(h \cdot \psi_{r(s)} \circ \varphi_{t}(a)\right)\right\| \\
\quad \leq\left\|\psi_{r(s)} \circ \varphi_{t}(g \cdot a)-h \cdot \psi_{r(s)} \circ \varphi_{t}(a)\right\|+\frac{1}{n}
\end{aligned}
$$

for all $g, h \in G_{n}, a, b \in K_{n}, s, t \in\left[0, t_{n+1}\right]$. We may assume that $s_{n}<s_{n+1}$ for all $n$ and that $\lim _{n \rightarrow \infty} s_{n}=\infty$. Let $v:[0, \infty) \rightarrow[0, \infty)$ be an increasing 
parametrization with $v\left(t_{n}\right)=s_{n}$. Let $w$ be any parametrization $w \geq v$, and consider any $a, b \in K_{n}, g, h \in G_{n}$. When $t \geq t_{m}, m \geq n$ and $s \geq t$, there is a $k \geq m$ such that $s \in\left[t_{k}, t_{k+1}\right]$. Then $w(s) \geq v(s) \geq v\left(t_{k}\right)=s_{k}$ and hence

$$
\begin{aligned}
\left\|\lambda_{w(s)} \circ \psi_{r(s)} \circ \varphi_{t}(g \cdot a)-h \cdot \lambda_{w(s)} \circ \psi_{r(s)} \circ \varphi_{t}(b)\right\| \\
\leq\left\|\lambda_{w(s)} \circ \psi_{r(s)} \circ \varphi_{t}(g \cdot a)-\lambda_{w(s)}\left(h \cdot \psi_{r(s)} \circ \varphi_{t}(b)\right)\right\| \\
\quad \quad \quad\left\|\lambda_{w(s)}\left(h \cdot \psi_{r(s)} \circ \varphi_{t}(b)\right)-h \cdot \lambda_{w(s)} \circ \psi_{r(s)} \circ \varphi_{t}(b)\right\| \\
\leq\left\|\psi_{r(s)} \circ \varphi_{t}(g \cdot a)-h \cdot \psi_{r(s)} \circ \varphi_{t}(b)\right\|+\frac{2}{k} \\
\leq \frac{2}{k}+\frac{1}{m}+\|g \cdot a-h \cdot b\| \leq \frac{3}{m}+\|g \cdot a-h \cdot b\|,
\end{aligned}
$$

for all $a, b \in K_{n}, g, h \in G_{n}$. This shows that the last condition holds for all parametrizations $w$ which are sufficiently large. The first conditions are met via similar, but slightly simpler arguments. Since $\lim _{t \rightarrow \infty}\left\|\mu_{t}(x)-\psi_{r(t)} \circ \varphi_{t}(x)\right\|=$ $0, x \in X$, we can make $w$ increase so rapidly that

$$
\lim _{t \rightarrow \infty}\left\|\lambda_{w(t)} \circ \mu_{t}(x)-\lambda_{w(t)} \circ \psi_{r(t)} \circ \varphi_{t}(x)\right\|=0, \quad x \in X .
$$

Finally, let $Y$ be a dense subset of $B$ which is a countable union of compact sets containing 0 such that $\bigcup_{t} \varphi_{t}(X) \subseteq Y$. We take $w$ such that $(Y, w)$ is a composition pair for $\lambda$ and $\psi_{r(\cdot)}$, in addition to all the other requirements. Then $[\lambda] \bullet([\psi] \bullet[\varphi])=[\beta]$ where $\beta: A \rightarrow D$ is an equicontinuous sequentially trivial asymptotic homomorphism such that

$\lim _{t \rightarrow \infty}\left\|\beta_{t}(x)-\lambda_{w(t)} \circ \mu_{t}(x)\right\|=\lim _{t \rightarrow \infty}\left\|\beta_{t}(x)-\lambda_{w(t)} \circ \psi_{r(t)} \circ \varphi_{t}(x)\right\|=0, \quad x \in X$.

Let $v: B \rightarrow D$ be an equicontinuous asymptotic homomorphism such that

$$
\lim _{t \rightarrow \infty}\left\|v_{t}(y)-\lambda_{w(t)} \circ \psi_{r(t)}(y)\right\|=0, \quad y \in Y .
$$

Let $(X, s)$ be a composition pair for $v$ and $\varphi$ such that $s(t) \geq t$ for all $t \in$ $[0, \infty)$. By using that $\bigcup_{t} \varphi_{t}(X) \subseteq Y$, we can choose the parametrization $s:[0, \infty) \rightarrow[0, \infty)$ such that

$$
\lim _{t \rightarrow \infty}\left\|v_{s(t)} \circ \varphi_{t}(x)-\lambda_{w(s(t))} \circ \psi_{r(s(t))} \circ \varphi_{t}(x)\right\|=0, \quad x \in X .
$$

Thanks to the special properties of $w$ we can construct homotopic equicontinuous asymptotic homomorphisms, $\lambda^{0}, \lambda^{1}: A \rightarrow D$, such that

$$
\lim _{t \rightarrow \infty}\left\|\lambda_{t}^{0}(x)-\lambda_{w(t)} \circ \psi_{r(t)} \circ \varphi_{t}(x)\right\|=0, \quad x \in X,
$$


and

$$
\lim _{t \rightarrow \infty}\left\|\lambda_{t}^{1}(x)-\lambda_{w(s(t))} \circ \psi_{r(s(t))} \circ \varphi_{t}(x)\right\|=0, \quad x \in X .
$$

The homotopy is given by an equicontinuous asymptotic homomorphism $\boldsymbol{\Phi}=$ $\left(\boldsymbol{\Phi}_{t}\right)$, where

$$
\lim _{t \rightarrow \infty} \sup _{\alpha \in[0,1]}\left\|\boldsymbol{\Phi}_{t}(x)(\alpha)-\lambda_{w(\alpha s(t)+(1-\alpha) t)} \circ \psi_{r(\alpha s(t)+(1-\alpha) t)} \circ \varphi_{t}(x)\right\|=0
$$

for all $x \in X . \Phi$ exists by the requirements we put on $w$. Note that the equicontinuity of $\lambda, \psi$ and $\boldsymbol{\Phi}$, combined with the sequential triviality of $\varphi$, implies that $\boldsymbol{\Phi}$ must be sequentially trivial. Since $\beta$ and $\lambda^{0}$ are asymptotically equal we conclude that $[\beta]=\left[\lambda^{1}\right]$ in $[[A, D]]_{0}$. Since $\lim _{t \rightarrow \infty} \| \lambda_{t}^{1}(x)-v_{s(t)}$ o $\varphi_{t}(x) \|=0, x \in X$, we have that $\left[\lambda^{1}\right]=[v] \bullet[\varphi]=\left([\lambda] \bullet\left[\psi_{r(\cdot)}\right]\right) \bullet[\varphi]=$ $([\lambda] \bullet[\psi]) \bullet[\varphi]$.

To get a pairing of $[[A, B]] \times[[B, C]]_{0} \rightarrow[[A, C]]_{0}$ we use the following definition.

Definition 3.7. A parametrization $r:[0, \infty) \rightarrow[0, \infty)$ is said to be invertible when $r$ is strictly increasing and $r(0)=0$. Let $A, B$ and $C$ be $G$ algebras, and let $\varphi=\left\{\varphi_{t}\right\}_{t \in[0, \infty)}: A \rightarrow B$ and $\psi=\left\{\psi_{t}\right\}_{t \in[0, \infty)}: B \rightarrow C$ be equicontinuous asymptotic homomorphisms. Assume that $\psi$ is sequentially trivial. A composition pair $(X, r)$ for $\varphi$ and $\psi$ is said to be invertible when $r$ is invertible and

$$
\lim _{t \rightarrow \infty} \sup _{\{n \in \mathrm{N}: n \geq r(t)\}}\left\|\psi_{n} \circ \varphi_{t}(a)\right\|=0
$$

for all $a \in X$.

By using that $\psi$ is sequentially trivial one sees that invertible composition pairs always exist. The crucial point about this notion is that when $(X, r)$ is an invertible composition pair,

$$
\lim _{n \rightarrow \infty} \psi_{n} \circ \varphi_{s^{-1}(n)}(x)=0
$$

for all $x \in X$ and all invertible parametrizations $s \geq r$.

Lemma 3.8. Let $\varphi: A \rightarrow B, \psi: B \rightarrow C$ be equicontinuous asymptotic homomorphisms. Assume that $\psi$ is sequentially trivial. Let $(X, r)$ be an invertible composition pair for $\psi$ and $\varphi$.

(a) For any invertible parametrization $s \geq r$ there is a sequentially trivial equicontinuous asymptotic homomorphism $\mu^{s}: A \rightarrow C$ such that

$$
\lim _{t \rightarrow \infty} \mu_{t}^{s}(x)-\psi_{t} \circ \varphi_{s^{-1}(t)}(x)=0, \quad x \in X .
$$


$\mu^{s}$ is unique up to asymptotic equality.

(b) When $s_{1}, s_{2}$ are invertible parametrizations such that $s_{i} \geq r, i=1,2$, the two sequentially trivial asymptotic homomorphisms $\mu^{s_{1}}$ and $\mu^{s_{2}}$ are homotopic.

Proof. (a) Construct $\lambda^{s}$ exactly as in the proof of (a) in Lemma 3.5. Set then

$$
\mu_{t}^{s}(a)=\lambda_{s^{-1}(t)}^{s}(a) .
$$

Then

$$
\lim _{t \rightarrow \infty}\left\|\mu_{t}^{s}(x)-\psi_{t} \circ \varphi_{s^{-1}(t)}(x)\right\|=0
$$

for all $x \in X$. Since $r$ satisfies (16) and $s \geq r$ it follows that $\lim _{n \rightarrow \infty} \mu_{n}^{s}(x)=0$ for all $x \in X$. By equicontinuity of $\mu^{s}$ and density of $X$ in $A$ it follows that $\mu^{s}$ is sequentially trivial.

(b) follows in the same way as (b) of Lemma 3.5. The only difference is that the homotopy $\boldsymbol{\Phi}$ is now obtained by setting

$$
\boldsymbol{\Psi}_{t}(x)(\alpha)=\psi_{t} \circ \varphi_{[\alpha s+(1-\alpha) r]^{-1}(t)}(x) .
$$

THEOREM 3.9. There is a map

$$
[[A, B]] \times[[B, C]]_{0} \ni(x, y) \mapsto y \bullet x \in[[A, C]]_{0}
$$

with the following properties:

(a) (Definition): When $\varphi: A \rightarrow B$ and $\psi: B \rightarrow C$ are equicontinuous asymptotic homomorphisms, with $\psi$ sequentially trivial, and $(X, r)$ is an invertible composition pair for $\psi$ and $\varphi$, then

$$
[\psi] \bullet[\varphi]=[\lambda],
$$

where $\lambda: A \rightarrow C$ is any sequentially trivial equicontinuous asymptotic homomorphism such that

$$
\lim _{t \rightarrow \infty}\left[\lambda_{t}(x)-\psi_{t} \circ \varphi_{s^{-1}(t)}(x)\right]=0, \quad x \in X,
$$

for some invertible parametrization $s \geq r$.

(b) (Associativity):

$$
z \bullet(y \bullet x)=(z \bullet y) \bullet x
$$

in $[[A, D]]_{0}$, when $x \in[[A, B]], y \in[[B, C]], z \in[[C, D]]_{0}$.

Proof. (a) follows in the same way as (a) of Theorem 3.6. One uses Lemma 3.8 instead of Lemma 3.5, of course. The proof of (b) is very similar to the proof of (b) of Theorem 3.6, and we leave the details to the reader. 


\section{Excision and Bott-periodicity}

In this section we describe how the pairings of Theorem 3.6 and Theorem 3.9 can be used to prove a series of fundamental properties of $[[A, B]]_{0}$. The methods are basically those developed in [3], [2], [5] and [8] and they all build on the Bott-periodicity theorem of Cuntz, [4]. In particular, the corresponding results involving $[[A, B]]$ instead of $[[A, B]]_{0}$ are all contained in [8]. Accordingly what we offer in this section is to a large extend merely a reading guide which shows how the known arguments can be adopted to deal with sequentially trivial asymptotic homomorphisms.

An extension

$$
0 \longrightarrow J \stackrel{j}{\longrightarrow} A \stackrel{p}{\longrightarrow} B \longrightarrow 0
$$

of $C^{*}$-algebras, where $A, B$ and $J$ are $G$-algebras, is said to be an extension of $G$-algebras when $j$ and $p$ are $G$-equivariant.

By using Lemma 1.4 of [10] the Connes-Higson construction from [3] can be used to obtain an asymptotic homomorphism $S B \rightarrow J$ from a given extension of $G$-algebras of the form (17), cf. Proposition 5.5 in [8].

THeORem 4.1. For any G-algebra $D$,

$$
[[D, S-]]_{0},
$$

is a functor from $G$-algebras to groups which is half-exact with respect to extensions of $G$-algebras.

Proof. Consider the $G$-extension (17), and let

$$
C_{p}=\{(a, f) \in A \oplus C B: f(1)=p(a)\}
$$

be the mapping cone of $p$, and let $\alpha: C_{p} \rightarrow A$ be the projection. Then

$$
\left[\left[D, C_{p}\right]\right]_{0} \stackrel{\alpha_{*}}{\longrightarrow}[[D, A]]_{0} \stackrel{p_{*}}{\longrightarrow}[[D, B]]_{0}
$$

is exact (as pointed sets). Indeed, if $\varphi: D \rightarrow A$ is a sequentially trivial asymptotic homomorphism such that $p_{*}[\varphi]=0$, there is a sequentially trivial asymptotic homomorphism $\boldsymbol{\Phi}: D \rightarrow I B$ such that $\pi_{0} \circ \boldsymbol{\Phi}_{t}=0$ and $\pi_{1} \circ \boldsymbol{\Phi}_{t}=$ $p \circ \varphi_{t}$ for all $t \in[0, \infty)$. Note that $\boldsymbol{\Phi}$ is a sequentially trivial asymptotic homomorphism $\boldsymbol{\Phi}: D \rightarrow C B=\{f \in I B: f(0)=0\}$ and that

$$
\psi_{t}(d)=\left(\varphi_{t}(d), \boldsymbol{\Phi}_{t}(d)\right),
$$

defines a sequentially trivial asymptotic homomorphism $\psi: D \rightarrow C_{p}$ such that $\alpha_{*}[\psi]=[\varphi]$. By applying (18) to the suspension of (17) we conclude that

$$
\left[\left[D, S C_{p}\right]\right]_{0} \stackrel{\alpha_{*}}{\longrightarrow}[[D, S A]]_{0} \stackrel{p_{*}}{\longrightarrow}[[D, S B]]_{0}
$$


is exact. By Proposition 5.14 of [8] $S C_{p}$ is equivalent to $S J$ in the category of homotopy classes of asymptotic homomorphisms. Therefore the pairing from Theorem 3.6 allows us to replace $S C_{p}$ with $S J$ in (19).

We now stabilize the functor. So let $\mathscr{K}$ denote the compact operators on an infinite-dimensional separable Hilbert space. For any $G$-algebra $A$ we consider $A \otimes \mathscr{K}$ as a $G$-algebra with the given $G$-action on $A$ tensored with the trivial action on $\mathscr{K}$. Set

$$
D(A, B)=[[S A \otimes \mathscr{K}, S B \otimes \mathscr{K}]]_{0} .
$$

Then $D$ is a bivariant functor from the category of $G$-algebras to the category of abelian groups. Since we consider $\mathscr{K}$ as a trivial $G$-algebra the standard proof shows that there is natural isomorphism $D(A, B) \simeq[[S A, S B \otimes \mathscr{K}]]_{0}$. More importantly, we also have Bott-periodicity in the form known from $K K$-theory and $E$-theory.

THEOREM 4.2 (Bott periodicity). There are natural isomorphisms,

$$
D\left(A, S^{2} B\right) \simeq D(A, B) \quad \text { and } \quad D\left(S^{2} A, B\right) \simeq D(A, B) .
$$

Furthermore, the suspension map $S: D(A, B) \rightarrow D(S A, S B)$ is an isomorphism.

Proof. By Proposition 6.16 of [8], $S^{3} \mathscr{K}$ and $S \mathscr{K}$ are equivalent in the category of homotopy classes of asymptotic homomorphisms. Hence the two isomorphisms, $D\left(A, S^{2} B\right) \simeq D(A, B)$ and $D\left(S^{2} A, B\right) \simeq D(A, B)$, are obtained by using Theorem 3.6 and Theorem 3.9, respectively. The fact that the suspension map is an isomorphism follows in the same way as in Propositions 6.16 and 6.17 of [8].

It follows from Theorem 4.1 that $D(A,-)$ is half-exact with respect to extensions of $G$-algebras. Similarly, by using Theorem 4.2 we can show that $D(-, B)$ is half-exact as a contravariant functor, but we are not going to need this fact.

Set

$$
E(A, B)=[[S A \otimes \mathscr{K}, S B \otimes \mathscr{K}]] .
$$

As in the case of $D$ we can remove $\mathscr{K}$ on the left, i.e. we have a natural

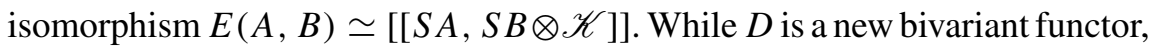
$E$ is only a slight generalization of the equivariant $E$-theory, denoted by $E_{G}$, of Guentner, Higson and Trout, [8]. To explain the relation, consider the Hilbert space

$$
H_{G}=\oplus_{i=1}^{\infty} L^{2}(G) .
$$


The direct sum of copies of the regular representation makes the $C^{*}$-algebra of compact operators on $H_{G}$ into a $G$-algebra which we denote by $\mathscr{K}_{G}$. Then

$$
E_{G}(A, B)=E\left(A \otimes \mathscr{K}_{G}, B \otimes \mathscr{K}_{G}\right) .
$$

\section{The six-term exact sequences}

Definition 5.1. A discrete asymptotic homomorphism $\varphi=\left(\varphi_{n}\right)_{n \in \mathrm{N}}: A \rightarrow$ $B$ is a sequence of maps $\varphi_{n}: A \rightarrow B, n \in \mathrm{N}$, such that

$$
\begin{aligned}
\lim _{n \rightarrow \infty}\left[\varphi_{n}(a b)-\varphi_{n}(a) \varphi_{n}(b)\right]=0, & a, b \in A, \\
\lim _{n \rightarrow \infty}\left[\varphi_{n}(a+\lambda b)-\varphi_{n}(a)-\lambda \varphi_{n}(b)\right]=0, & a, b \in A, \quad \lambda \in \mathrm{C}, \\
\lim _{n \rightarrow \infty}\left[\varphi_{n}\left(a^{*}\right)-\varphi_{n}(a)^{*}\right]=0, & a \in A . \\
\lim _{n \rightarrow \infty}\left[\varphi_{n}(g \cdot a)-g \cdot \varphi_{n}(a)\right]=0, & g \in G, \quad a \in A .
\end{aligned}
$$

Definition 5.2. Two discrete asymptotic homomorphisms, $\varphi=\left(\varphi_{n}\right)_{n \in \mathbb{N}}$ : $A \rightarrow B$ and $\psi=\left(\psi_{n}\right)_{n \in \mathrm{N}}$ are homotopic when there is a discrete asymptotic homomorphism $\boldsymbol{\Phi}=\left(\boldsymbol{\Phi}_{n}\right)_{n \in \mathrm{N}}: A \rightarrow I B$ such that $\pi_{0} \circ \boldsymbol{\Phi}_{n}(a)=\varphi_{n}(a)$ and $\pi_{1} \circ \boldsymbol{\Phi}_{n}(a)=\psi_{n}(a)$ for all $n \in \mathbf{N}, a \in A$.

The set of homotopy classes of discrete asymptotic homomorphisms from $A$ to $B$ will be denoted by $[[A, B]]_{\mathrm{N}}$. Then $[[A, S B]]_{\mathrm{N}}$ is a group, $\left[\left[A, S^{2} B\right]\right]_{\mathrm{N}}$ is an abelian group, and $[[A, S B]]_{N}$ is abelian when $B$ is stable.

In order to relate discrete asymptotic homomorphisms to sequentially trivial asymptotic homomorphisms, we need the following lemma.

Lemma 5.3. Let $\varphi: A \rightarrow B$ be a sequentially trivial asymptotic homomorphism. There is then a sequence $\left\{\delta_{n}\right\}$ in $] 0,1[$ and a sequentially trivial asymptotic homomorphism $\psi: A \rightarrow B$ such that

(1) $\psi_{t}=0, t \in\left[n-\delta_{n}, n+\delta_{n}\right]$,

(2) $\lim _{t \rightarrow \infty} \varphi_{t}(a)-\psi_{t}(a)=0, a \in A$.

Proof. By Proposition 2.4 we may assume that $\varphi$ is equicontinuous. Let $a_{1}, a_{2}, a_{3}, \ldots$, be a dense sequence in $A$. For each $n \in \mathrm{N}$, choose $\left.\delta_{n} \in\right] 0,1[$ such that

$$
\left\|\varphi_{t}\left(a_{i}\right)\right\| \leq\left\|\varphi_{n}\left(a_{i}\right)\right\|+\frac{1}{n}, \quad i=1,2, \ldots, n, \quad t \in\left[n-2 \delta_{n}, n+2 \delta_{n}\right] .
$$

Let $f:[0, \infty) \rightarrow[0,1]$ be a continuous function such that $f(t)=1, t \notin$ $\bigcup_{n}\left[n-2 \delta_{n}, n+2 \delta_{n}\right]$ and $f(t)=0, t \in \bigcup_{n}\left[n-\delta_{n}, n+\delta_{n}\right]$. Set $\psi_{t}(a)=$ 
$f(t) \varphi_{t}(a)$. Then $\lim _{t \rightarrow \infty} \psi_{t}(a)-\varphi_{t}(a)=0$ for all $a \in\left\{a_{1}, a_{2}, a_{3}, \ldots\right\}$, and by equicontinuity the same is true for all $a \in A$.

Let $\varphi=\left(\varphi_{n}\right)_{n \in \mathrm{N}}: A \rightarrow S B=C_{0}(0,1) \otimes B$ be a discrete asymptotic homomorphism. For each $t \in[0, \infty)$, define $\alpha(\varphi)_{t}: A \rightarrow B$ by

$$
\alpha(\varphi)_{t}(a)=\varphi_{n}(a)(t-n), \quad t \in[n, n+1] .
$$

It is straightforward to check that $\alpha(\varphi)=\left(\alpha(\varphi)_{t}\right)_{t \in[0, \infty)}$ is a sequentially trivial asymptotic homomorphism.

Lemma 5.4. The map $[\varphi] \mapsto[\alpha(\varphi)]$ is a bijection $\alpha:[[A, S B]]_{\mathrm{N}} \rightarrow$ $[[A, B]]_{0}$.

Proof. It is easy to see that the map is well-defined. Let $\varphi: A \rightarrow B$ be a sequentially trivial asymptotic homomorphism. By Lemma $5.3[\varphi] \in[[A, B]]_{0}$ is also represented by a sequentially trivial asymptotic homomorphism $\psi$ which satisfies that $\psi_{n}=0$ for all $n \in \mathrm{N}$. Define, for each $n \in \mathrm{N}, \lambda_{n}: A \rightarrow S B$ by $\left.\lambda_{n}(a)(t)=\psi_{n+t}(a), t \in\right] 0,1[$. Then $\lambda$ is a discrete asymptotic homomorphism such that $\alpha(\lambda)=\psi$. This proves the surjectivity of $\alpha$. To prove the injectivity, let $\varphi, \psi: A \rightarrow S B$ be discrete asymptotic homomorphisms, and let $\boldsymbol{\Phi}: A \rightarrow I B$ be a sequentially trivial asymptotic homomorphism such that $\pi_{1} \circ \boldsymbol{\Phi}_{t}(a)=\alpha(\varphi)_{t}(a), \pi_{0} \circ \boldsymbol{\Phi}_{t}(a)=\alpha(\psi)_{t}(a), a \in A, t \in[0, \infty)$. By Lemma 5.3 and the preceding argument we can find a discrete asymptotic homomorphism $\boldsymbol{\Psi}: A \rightarrow I S B$ such that $\lim _{t \rightarrow \infty} \alpha(\boldsymbol{\Psi})_{t}(a)-\boldsymbol{\Phi}_{t}(a)=$ 0 for all $a \in A$. It follows that $\lim _{n \rightarrow \infty} \pi_{0} \circ \boldsymbol{\Psi}_{n}(a)-\psi_{n}(a)=0$ and $\lim _{n \rightarrow \infty} \pi_{1} \circ \boldsymbol{\Psi}_{n}(a)-\varphi_{n}(a)=0$ for all $a \in A$. Thus $\varphi$ and $\psi$ are homotopic to $\pi_{1} \circ \boldsymbol{\Psi}$ and $\pi_{0} \circ \boldsymbol{\Psi}$, respectively, and hence also to each other.

Combining the bijection $\alpha$ with the obvious (forgetful) map $[[A, B]]_{0} \rightarrow$ $[[A, B]]$, we get a map

$$
c_{0}:[[A, S B]]_{\mathrm{N}} \rightarrow[[A, B]] .
$$

We seek the kernel of $c_{0}$, i.e. the set of elements in $x \in[[A, S B]]_{N}$ such that $c_{0}(x)=0$ in $[[A, B]]$. To this end we introduce an automorphism $\sigma$ of $[[A, S B]]_{\mathrm{N}}$. When $\varphi=\left(\varphi_{n}\right)_{n \in \mathrm{N}}$ is a discrete asymptotic homomorphism we define a new discrete asymptotic homomorphism, $\sigma(\varphi)$, by $\sigma(\varphi)_{n}=\varphi_{n+1}$. It is clear that we get a group automorphism, $\sigma$, of $[[A, S B]]_{\mathrm{N}}$ by defining $\sigma[\varphi]=[\sigma(\varphi)]$.

Lemma 5.5. Let $x \in[[A, S B]]_{\mathrm{N}}$. Then $c_{0}(x)=0$ in $[[A, B]]$ if and only if there is a $y \in[[A, S B]]_{\mathrm{N}}$ such that $x=y \sigma(y)^{-1}$. 
Proof. Let $\varphi=\left(\varphi_{n}\right)_{n \in \mathrm{N}}$ be a discrete asymptotic homomorphism. Then $c_{0}\left([\varphi][\sigma(\varphi)]^{-1}\right)$ is represented by an asymptotic homomorphism $\psi=$ $\left(\psi_{t}\right)_{t \in[0, \infty)}$ such that

$$
\psi_{n-s}=\psi_{n+s}, \quad n \in \mathbf{N}, \quad s \in\left[0, \frac{1}{2}\right]
$$

and $\psi_{n+\frac{1}{2}}=0, n \in \mathrm{N}$. For each $\lambda \in[0,1]$, define $\kappa_{\lambda}:[0, \infty) \rightarrow[0, \infty)$ by

$$
\kappa_{\lambda}(t)= \begin{cases}\lambda t+\left(n-\frac{1}{2}\right)(1-\lambda), & t \in\left[n-\frac{1}{2}, n[\right. \\ \lambda t+\left(n+\frac{1}{2}\right)(1-\lambda), & t \in\left[n, n+\frac{1}{2}\right] .\end{cases}
$$

Although $\kappa_{\lambda}$ is not continuous when $\lambda \neq 1$, we can, thanks to (24), define an asymptotic homomorphism $\boldsymbol{\Phi}: A \rightarrow I B$ by $\boldsymbol{\Phi}_{t}(a)(\lambda)=\psi_{\kappa_{\lambda}(t)}(a), \lambda \in[0,1]$. Then $\pi_{1} \circ \boldsymbol{\Phi}=\psi$ and $\pi_{0} \circ \boldsymbol{\Phi}=0$, showing that $c_{0}\left([\varphi][\sigma(\varphi)]^{-1}\right)=0$.

Conversely, let $\varphi=\left(\varphi_{n}\right)_{n \in \mathrm{N}}$ be a discrete asymptotic homomorphism such that $c_{0}[\varphi]=0$. This means that there is an asymptotic homomorphism $\boldsymbol{\Phi}$ : $A \rightarrow I B$ such that $\pi_{1} \circ \boldsymbol{\Phi}=0, \pi_{0} \circ \boldsymbol{\Phi}=\alpha(\varphi)$. For each $s \in[0,1], t \in[0, \infty)$, define $\boldsymbol{\Phi}(t, s): A \rightarrow B$ by $\boldsymbol{\Phi}(t, s)(a)=\pi_{s} \circ \boldsymbol{\Phi}_{t}(a)$. Define $\psi_{n}: A \rightarrow S B$ by $\psi_{n}(a)(t)=\boldsymbol{\Phi}(n, t)$. Since $\boldsymbol{\Phi}$ is an asymptotic homomorphism we see that $\psi=\left(\psi_{n}\right)_{n \in \mathrm{N}}$ is a discrete asymptotic homomorphism. We claim that $[\varphi]=[\psi][\sigma(\psi)]^{-1}$. To see this, choose continuous maps $\Lambda_{n}:[n, n+1] \times$ $[0,1] \rightarrow[n, n+1] \times[0,1]$ such that the following hold:

(1) $\Lambda_{n}(n, y)=(n, 0), y \in[0,1]$,

(2) $\Lambda_{n}(n+1, y)=(n+1,0), y \in[0,1]$,

(3) $\Lambda_{n}(x, 0)=(x, 0), x \in[0,1]$,

(4) $\Lambda_{n}\left(n+\frac{1}{3}, 1\right)=(n, 1)$,

(5) $\Lambda_{n}\left(n+\frac{2}{3}, 1\right)=(n+1,1)$,

(6) $\Lambda_{n}$ maps $\left\{(x, 1): x \in\left[n, n+\frac{1}{3}\right]\right\}$ homeomorphically onto $\{(n, y)$ : $y \in[0,1]\}$,

(7) $\Lambda_{n}$ maps $\left\{(x, 1): x \in\left[n+\frac{1}{3}, n+\frac{2}{3}\right]\right\}$ homeomorphically onto $\{(x, 1)$ : $x \in[0,1]\}$,

(8) $\Lambda_{n}$ maps $\left\{(x, 1): x \in\left[n+\frac{2}{3}, n+1\right]\right\}$ homeomorphically onto $\{(n+$ $1, y): y \in[0,1]\}$.

Define $\boldsymbol{\Psi}_{n}: A \rightarrow I S B$ by $\boldsymbol{\Psi}_{n}(a)(s, t)=\boldsymbol{\Phi}\left(\Lambda_{n}(n+t, s)\right)(a), s \in[0,1], t \in$ $(0,1)$. Then $\boldsymbol{\Psi}=\left(\boldsymbol{\Psi}_{n}\right)_{n \in \mathrm{N}}$ is a discrete asymptotic homomorphism giving us a homotopy between $\varphi: A \rightarrow S B$ and a discrete asymptotic homomorphism 
$\lambda=\left(\lambda_{n}\right)_{n \in \mathrm{N}}: A \rightarrow S B$ such that

$$
\lambda_{n}(a)(t)= \begin{cases}\psi_{n}(a)\left(\mu_{+}(t)\right), & t \in\left(0, \frac{1}{3}\right) \\ 0, & t \in\left[\frac{1}{3}, \frac{2}{3}\right], \\ \psi_{n+1}(a)\left(\mu_{-}(t)\right), & t \in\left(\frac{2}{3}, 1\right)\end{cases}
$$

where $\mu_{+}:\left[0, \frac{1}{3}\right] \rightarrow[0,1]$ is a homeomorphism homotopic to $t \mapsto 3 t$, and $\mu_{-}:\left[\frac{2}{3}, 1\right] \rightarrow[0,1]$ is a homeomorphism homotopic to $t \mapsto-3 t+3$. Since $\lambda$ is clearly homotopic to $[\psi][\sigma(\psi)]^{-1}$, this completes the proof.

When $\varphi=\left(\varphi_{t}\right)_{t \in[0, \infty)}: A \rightarrow B$ is an asymptotic homomorphism we let $\left.\varphi\right|_{\mathrm{N}}$ denote the discrete asymptotic homomorphism $\left(\varphi_{n}\right)_{n \in \mathrm{N}}$. We can then define a map $d_{0}:[[A, B]] \rightarrow[[A, B]]_{\mathrm{N}}$ such that $d_{0}[\varphi]=\left[\left.\varphi\right|_{\mathrm{N}}\right]$.

Lemma 5.6. The sequence

$$
[[A, S B]]_{\mathrm{N}} \stackrel{c_{0}}{\longrightarrow}[[A, B]] \stackrel{d_{0}}{\longrightarrow}[[A, B]]_{\mathrm{N}}^{\sigma} \longrightarrow 0
$$

is an exact sequence of pointed sets.

Proof. $d_{0}([[A, B]]) \subseteq[[A, B]]_{\mathrm{N}}^{\sigma}$ : Let $\varphi=\left(\varphi_{t}\right)_{t \in[0, \infty)}$ be an asymptotic homomorphism. Define $\boldsymbol{\Phi}_{n}: A \rightarrow I B$ by $\boldsymbol{\Phi}_{n}(a)(t)=\varphi_{n+t}(a)$. Then $\boldsymbol{\Phi}=\left(\boldsymbol{\Phi}_{n}\right)_{n \in \mathbf{N}}: A \rightarrow I B$ is a discrete asymptotic homomorphism defining a homotopy between $d_{0}[\varphi]$ and $\sigma\left(d_{0}[\varphi]\right)$.

$[[A, B]]_{\mathrm{N}}^{\sigma} \subseteq d_{0}([[A, B]]):$ Let $\varphi=\left(\varphi_{n}\right)_{n \in \mathrm{N}}: A \rightarrow B$ be a discrete asymptotic homomorphism such that $[\varphi]=\sigma[\varphi]$. There is then a discrete asymptotic homomorphism $\boldsymbol{\Phi}=\left(\boldsymbol{\Phi}_{n}\right)_{n \in \mathrm{N}}: A \rightarrow I B$ such that $\pi_{0} \circ \boldsymbol{\Phi}_{n}=\varphi_{n}$ and $\pi_{1} \circ \boldsymbol{\Phi}_{n}=\varphi_{n+1}$. Define an asymptotic homomorphism $\psi=\left(\psi_{t}\right)_{t \in[0, \infty)}$ : $A \rightarrow B$ by $\psi_{t}(a)=\boldsymbol{\Phi}_{n}(a)(t-n), t \in[n, n+1]$. Then $d_{0}[\psi]=[\varphi]$.

It is clear that $c_{0}\left([[A, S B]]_{\mathrm{N}}\right) \in \operatorname{ker} d_{0}$. To prove that $\operatorname{ker} d_{0} \subseteq c_{0}\left([[A, S B]]_{\mathrm{N}}\right)$, let $\varphi=\left(\varphi_{t}\right)_{t \in[0, \infty)}: A \rightarrow B$ be an asymptotic homomorphism such that $d_{0}[\varphi]=0$. There is then a discrete asymptotic homomorphism $\boldsymbol{\Phi}=\left(\boldsymbol{\Phi}_{n}\right)_{n \in \mathrm{N}}$ : $A \rightarrow I B$ such that $\pi_{0} \circ \boldsymbol{\Phi}_{n}=0$ and $\pi_{1} \circ \boldsymbol{\Phi}_{n}=\varphi_{n}$ for all $n \in \mathbf{N}$. For each $n \in \mathrm{N}$, set $\Delta_{n}=[n, n+1] \times\{1\} \cup\{n\} \times[0,1] \cup\{n+1\} \times[0,1]$ which is a subset of $[n, n+1] \times[0,1]$. For each $a \in A$ we define a continuous function $\Psi_{n}(a): \Delta_{n} \rightarrow B$ by

$$
\boldsymbol{\Psi}_{n}(a)(t, s)= \begin{cases}\varphi_{t}(a), & (t, s) \in[n, n+1] \times\{1\} \\ \boldsymbol{\Phi}_{n}(a)(s), & (t, s) \in\{n\} \times[0,1] \\ \boldsymbol{\Phi}_{n+1}(a)(s), & (t, s) \in\{n+1\} \times[0,1]\end{cases}
$$


Let $\Lambda_{n}:[n, n+1] \times[0,1] \rightarrow \Delta_{n}$ be a continuous retraction, and define a function $\boldsymbol{\Psi}_{t}(a): A \rightarrow I B$ by $\boldsymbol{\Psi}_{t}(a)(s)=\boldsymbol{\Psi}_{n}(a)\left(\Lambda_{n}(t, s)\right), t \in[n, n+1]$, $s \in[0,1]$. Then $\boldsymbol{\Psi}=\left(\boldsymbol{\Psi}_{t}\right)_{t \in[0, \infty)}: A \rightarrow I B$ is an asymptotic homomorphism giving us a homotopy connecting $\varphi$ to a sequentially trivial asymptotic homomorphism.

By using Lemma 5.4 we identify $D(A, B)$ with $\left[\left[S A \otimes \mathscr{K}, S^{2} B \otimes \mathscr{K}\right]\right]_{\mathrm{N}}$. In this way $\sigma$ defines a group automorphism of $D(A, B)$, and the map $c_{0}$ becomes the obvious (forgetful) map $c: D(A, B) \rightarrow E(A, B) . d_{0}$ becomes a map $d=\alpha^{-1} \circ d_{0}: E(A, S B) \rightarrow D(A, B)$. Let finally $B: E(A, B) \rightarrow$ $E\left(A, S^{2} B\right)$ be the Bott-isomorphism.

Theorem 5.7. The sequence

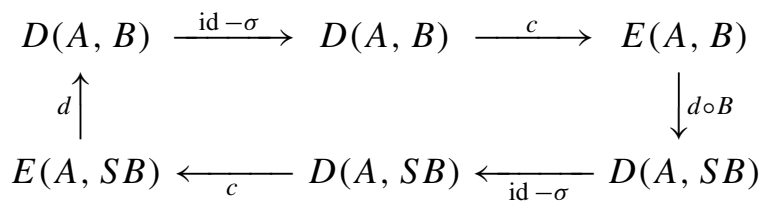

is exact.

Proof. By Lemma 5.5 and Lemma 5.6 it suffices to prove exactness at $E(A, B)$. But this follows from Lemma 5.6 and the trivial observation that the diagram

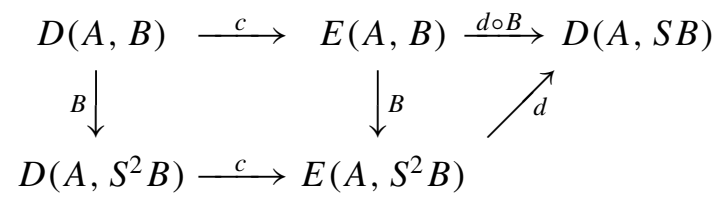

commutes.

It is clear that there is also a dual version of the six-terms exact sequence of Theorem 5.7. All one has to do is to use suspension and Bott-periodicity (in the second variable) to translate the sequence of Theorem 5.7 to another where it is the first variable which becomes suspended and de-suspended. To describe how the maps are changed, let $\varphi=\left(\varphi_{n}\right)_{n \in \mathrm{N}}: A \rightarrow B$ be a discrete asymptotic homomorphism. Define an asymptotic homomorphism $c^{\prime}(\varphi): S A \rightarrow B$ by $c^{\prime}(\varphi)_{t}(f)=\varphi_{n}(f(t-n)), t \in[n, n+1] . c^{\prime}$ gives us a map $c^{\prime}:[[A, B]]_{\mathrm{N}} \rightarrow[[S A, B]]_{0}$. The composition

$$
[[A, B]] \stackrel{d_{0}}{\longrightarrow}[[A, B]]_{\mathrm{N}} \stackrel{c^{\prime}}{\longrightarrow}[[S A, B]]_{0}
$$

will be denoted by $d^{\prime}$. Then $d^{\prime}$ gives rise to a map $d^{\prime}: E(A, B) \rightarrow D(S A, B)$. 
THEOREM 5.8. The sequence

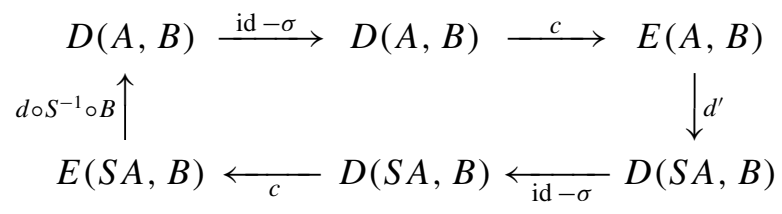

is exact.

Proof. In order to transform the diagram of Theorem 5.7 to get the dual version, observe that the following two diagrams commute :

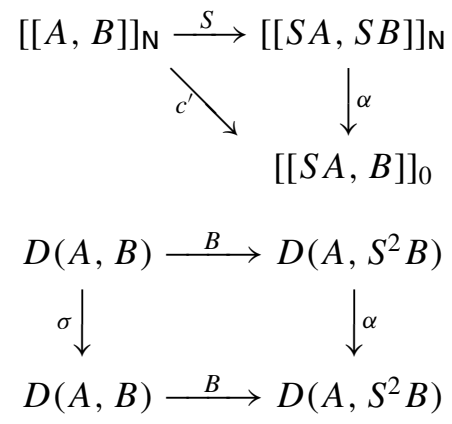

The first diagram is seen to commute by checking on a simple tensor $g \otimes a$, $g \in S, a \in A$. As for the last diagram, it suffices to remember that the Bottisomorphism $D(A, B) \rightarrow D\left(A, S^{2} B\right)$ is induced by a genuine (equivariant) *-homomorphism $S B \otimes \mathscr{K} \rightarrow S^{3} B \otimes \mathscr{K}$. With the commutativity of these two diagrams established, the rest is merely a little diagram chasing which we leave to the reader.

Notice that Lemma 5.4 shows that $[[A, B]]_{0}$ has the structure of a group. The automorphism $\sigma$ of $[[A, S B]]_{\mathrm{N}}$ corresponds to the automorphism (again denoted by $\sigma$ ) on $[[A, B]]_{0}$ which is given by $\sigma(\varphi)_{t}=\varphi_{t+1}$.

We have the following lemma which removes a couple of redundant suspensions and will be used in the next section.

Lemma 5.9. There is a natural isomorphism

$$
D(A, S B)^{\sigma} \simeq[[S A, S B \otimes \mathscr{K}]]_{\mathrm{N}}^{\sigma} .
$$

Proof. The Bott-isomorphism $B$ gives us a commuting diagram

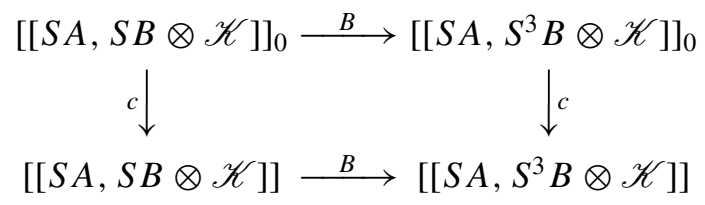


By Lemma 5.6 this diagram can be completed to give us a natural isomorphism

$$
[[S A, S B \otimes \mathscr{K}]]_{\mathrm{N}}^{\sigma} \simeq\left[\left[S A, S^{3} B \otimes \mathscr{K}\right]\right]_{\mathrm{N}}^{\sigma} .
$$

Since $\left[\left[S A, S^{3} B \otimes \mathscr{K}\right]\right]_{N}^{\sigma} \simeq D(A, S B)^{\sigma}$, this completes the proof.

\section{KK-theory}

A (discrete) asymptotic homomorphism will be called completely positive when the individual maps are completely positive linear equivariant contractions. By imposing the condition of complete positivity on the homotopies we obtain the sets $[[A, B]]_{c p}$ and $[[A, B]]_{\mathrm{N}, c p}$, of homotopy classes of completely positive asymptotic homomorphisms from $A$ to $B$, and discrete completely positive asymptotic homomorphisms from $A$ to $B$, respectively. Similarly, we let $[[A, B]]_{0, c p}$ denote the homotopy classes of sequentially trivial completely positive asymptotic homomorphisms. The composition products and pairings between $[[\cdot, \cdot]]_{c p}$ and $[[\cdot, \cdot]]_{0, c p}$ can then be defined in a similar, but simpler way as in Section 3. And the proof of Lemma 5.4 allows us to identify $[[A, S B]]_{\mathrm{N}, c p}$ with $[[A, B]]_{0, c p}$. However, to have the Connnes-Higson construction available we need to stabilize and saturate the $G$-algebras by tensoring with the $G$-algebra $\mathscr{K}_{G}$ which was introduced at the end of Section 4 to compare $E$ and $E_{G}$. By tensoring all $G$-algebras by $\mathscr{K}_{G}$ we come in a situation where the Connes-Higson construction out of an extension of $G$-algebras with a completely positive contractive and equivariant section for the quotient map produces an asymptotic homomorphism which is not only completely positive, but also equivariant, cf. Lemma 3.3 and Lemma 4.4 of [17]. As a result we find that

$$
\left[\left[S A \otimes \mathscr{K}_{G}, S B \otimes \mathscr{K}_{G}\right]\right]_{c p}=K K^{G}(A, B),
$$

cf. Theorem 4.9 of [17]. In this way the six-term exact sequences of Theorem 5.7 and Theorem 5.8 turn into the following two, where

$$
\begin{aligned}
K J^{G}(A, B) & =\left[\left[S A \otimes \mathscr{K}_{G}, S^{2} B \otimes \mathscr{K}_{G}\right]\right]_{\mathrm{N}, c p} \\
& =\left[\left[S A \otimes \mathscr{K}_{G}, S B \otimes \mathscr{K}_{G}\right]\right]_{0, c p} .
\end{aligned}
$$

THEOREM 6.1. The following two sequences are exact.

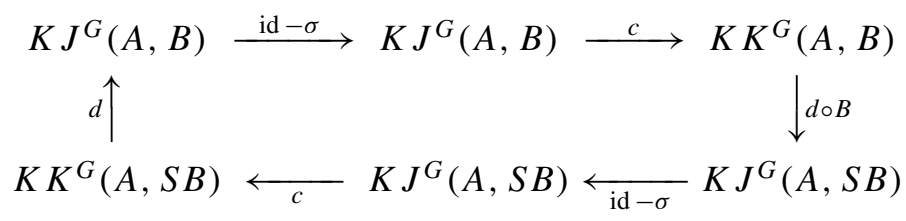




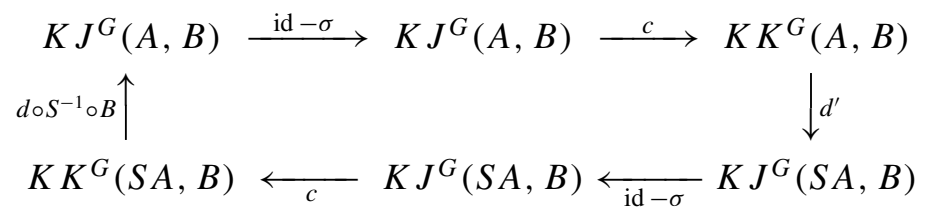

To see in which way these exact sequences are related to the UCT, let us restrict attention to the non-equivariant case, i.e. the case $G=0$. In this case the Connes-Higson construction gives us an isomorphism

$$
C H: \operatorname{Ext}^{-1}(A, B) \rightarrow[[S A, B \otimes \mathscr{K}]]_{c p} .
$$

This was pointed out in [12], but is really something which follows from [11], [7] and [9]. Let us assume that $A$ is in the Bootstrap-category for which the UCT holds, cf. [13], [6]. Then $\operatorname{Pext}\left(K_{*}(A), K_{*-1}(B)\right)$ can be realized as a subgroup of $\operatorname{Ext}^{-1}(S A, B)$, and it was shown in [12] that the isomorphism $\mathrm{CH}$ takes this subgroup onto the range of

$$
c:\left[\left[S^{2} A \otimes \mathscr{K}, B \otimes \mathscr{K}\right]\right]_{0, c p} \rightarrow\left[\left[S^{2} A \otimes \mathscr{K}, B \otimes \mathscr{K}\right]\right]_{c p} \simeq K K(A, B),
$$

i.e. onto the subgroup of $\left[\left[S^{2} A \otimes \mathscr{K}, B \otimes \mathscr{K}\right]\right]_{c p}$ consisting of the elements which may be represented by a sequentially trivial asymptotic homomorphism. It follows from this, the exactness of the diagram(s) in Theorem 6.1 and the version of the UCT from [6], that the range of the map $d \circ B$ in Theorem 6.1 can be identified with $K L(A, B)$. In other words, by unsplicing the first six-term exact sequence of Theorem 6.1 we obtain an extension of groups which is the same as the extension in the UCT theorem, in the form it was given in [6]. And by using the version of Lemma 5.9 which involves completely positive asymptotic homomorphisms, this leads us to the conclusion that

$$
K L(A, B) \simeq K J(A, S B)^{\sigma} \simeq[[S A, S B \otimes \mathscr{K}]]_{\mathrm{N}, c p}^{\sigma} .
$$

\section{Unsuspending D-theory}

In order to keep our promises from the introduction we must show that $D(A, B)$ $\simeq[[S A, B \otimes \mathscr{K}]]_{\mathrm{N}}$. The argument for this is a repetition of the arguments of Dadarlat and Loring from [7] once we have obtained the following pairing.

A discrete asymptotic homomorphism $\varphi=\left(\varphi_{n}\right)_{n \in \mathbb{N}}: A \rightarrow B$ is called equicontinuous when the obvious analogues of (6) and (7) hold. In analogy with Lemma 2.6 we have that the set of homotopy classes of discrete asymptotic homomorphisms is the same as the set of homotopy classes of equicontinuous discrete asymptotic homomorphisms. This is helpful in the construction of a pairing $[[A, B]]_{\mathrm{N}} \times[[B, C]] \rightarrow[[A, C]]_{\mathrm{N}}$ in the same way as equicontinuity was helpful in Section 3. 
Definition 7.1. Let $A, B$ and $C$ be $G$-algebras. Let $\varphi=\left\{\varphi_{n}\right\}: A \rightarrow B$ be an equicontinuous discrete asymptotic homomorphism and $\psi=\left\{\psi_{t}\right\}_{t \in[0, \infty)}$ : $B \rightarrow C$ an equicontinuous asymptotic homomorphism. A composition pair for $\psi$ and $\varphi$ is a dense subset $X \subseteq A$ which is the union of a sequence of compact subsets containing 0 and a parametrization $r:[0, \infty) \rightarrow[0, \infty)$ such that

$$
\lim _{n \rightarrow \infty} \sup _{s \geq r(n)}\left\|\psi_{s} \circ \varphi_{n}(a b)-\psi_{s} \circ \varphi_{n}(a) \psi_{s} \circ \varphi_{n}(b)\right\|=0
$$

$$
\begin{gathered}
\lim _{n \rightarrow \infty} \sup _{s \geq r(n)}\left\|\psi_{s} \circ \varphi_{n}(a+\lambda b)-\psi_{s} \circ \varphi_{n}(a)-\lambda \psi_{s} \circ \varphi_{n}(b)\right\|=0, \\
\lim _{n \rightarrow \infty} \sup _{s \geq r(n)}\left\|\psi_{s} \circ \varphi_{n}\left(a^{*}\right)-\psi_{s} \circ \varphi_{n}(a)^{*}\right\|=0,
\end{gathered}
$$

for all $a, b \in X, \lambda \in \mathrm{C}$, and for every compact subset $K \subseteq G$, every pair $a, b \in X$ and every $\epsilon>0$ there is a $n_{0} \in \mathrm{N}$ such that

$$
\sup _{s \geq r(n)}\left\|g \cdot \psi_{s} \circ \varphi_{n}(a)-\psi_{s} \circ \varphi_{n}(h \cdot b)\right\| \leq\|g \cdot a-h \cdot b\|+\epsilon,
$$

for all $g, h \in K$ when $n \geq n_{0}$.

We can then repeat the proof of Theorem 3.6 to obtain the following.

THEOREM 7.2. There is a map

$$
[[A, B]]_{\mathrm{N}} \times[[B, C]] \ni(x, y) \mapsto y \bullet x \in[[A, C]]_{\mathrm{N}}
$$

with the following properties:

(a) (Definition): When $\varphi: A \rightarrow B$ is an equicontinuous discrete asymptotic homomorphism and $\psi: B \rightarrow C$ an equicontinuous asymptotic homomorph$i s m$, and $(X, r)$ is a composition pair for $\psi$ and $\varphi$, then

$$
[\psi] \bullet[\varphi]=[\lambda]
$$

where $\lambda: A \rightarrow C$ is any equicontinuous discrete asymptotic homomorphism such that

$$
\lim _{n \rightarrow \infty}\left[\lambda_{n}(x)-\psi_{s(n)} \circ \varphi_{n}(x)\right]=0, \quad x \in X,
$$

for some parametrization $s \geq r$.

(b) (Associativity):

$$
z \bullet(y \bullet x)=(z \bullet y) \bullet x,
$$

in $[[A, D]]_{\mathrm{N}}$, when $x \in[[A, B]]_{\mathrm{N}}, y \in[[B, C]], z \in[[C, D]]$. 
By using this pairing it follows that $[[S A,-\otimes \mathscr{K}]]_{N}$ is a split-exact functor from the category of $G$-algebras to the category of abelian groups for any $G$ algebra $A$, cf. Proposition 3.2 of [7]. ${ }^{1}$

Lemma 7.3. The suspension map $S:[[S A, B \otimes \mathscr{K}]]_{N} \rightarrow\left[\left[S^{2} A, S B \otimes\right.\right.$ $\mathscr{K}]]_{\mathrm{N}}$ is an isomorphism.

Proof. The standard proof shows that $[[S A, B \otimes \mathscr{K}]]_{\mathrm{N}} \simeq[[S A \otimes \mathscr{K}, B \otimes$ $\mathscr{K}]]_{\mathrm{N}}$. It suffices therefore to show that $S:[[S A \otimes \mathscr{K}, B \otimes \mathscr{K}]]_{\mathrm{N}} \rightarrow\left[\left[S^{2} A \otimes\right.\right.$ $\mathscr{K}, S B \otimes \mathscr{K}]]_{\mathrm{N}}$ is an isomorphism. This follows now as in [7]. The inverse of $S$ is given by

$$
[\varphi] \mapsto[\alpha] \bullet[(S \varphi) \circ \beta],
$$

where $\alpha: S^{2} B \otimes \mathscr{K} \rightarrow B \otimes \mathscr{K}$ is an asymptotic homomorphism and $\beta$ : $S A \otimes \mathscr{K} \rightarrow S^{3} A \otimes \mathscr{K}$ is a $*$-homomorphism.

THEOREM 7.4. $D(A, B) \simeq[[S A, B \otimes \mathscr{K}]]_{\mathrm{N}}$.

Proof. By Lemma 7.3 we have that $[[S A, B \otimes \mathscr{K}]]_{N} \simeq\left[\left[S^{3} A \otimes \mathscr{K}, S^{2} B \otimes\right.\right.$ $\mathscr{K}]]_{\mathrm{N}}$. By Lemma 5.4 the latter group is $D\left(S^{2} A, B\right)$ and this is the same as $D(A, B)$ by Theorem 4.2 .

By restricting attention to completely positive asymptotic homomorphisms we get of course a similar de-suspension of the functor $K J^{G}(A, B)$ from Section 6:

$$
K J^{G}(A, B) \simeq[[S A, B \otimes \mathscr{K}]]_{\mathrm{N}, c p} .
$$

REMARK 7.5. Most, if not all, of the tools available for the calculation of $K K(A, B)$ can also be used for calculating $D(A, B)$, at least in the nonequivariant case where $G=0$. To illustrate this, note first that it is easy to see that $D(\mathrm{C}, B)=\left[\left[C_{0}(\mathrm{R}), B \otimes \mathscr{K}\right]\right]_{\mathrm{N}}=\prod_{n=1}^{\infty} K_{1}(B) / \oplus_{n=1}^{\infty} K_{1}(B)$. Hence, by Bott-periodicity, $D\left(C_{0}(\mathrm{R}), B\right)=\prod_{n=1}^{\infty} K_{0}(B) / \oplus_{n=1}^{\infty} K_{0}(B)$. Since $D(-, B)$ has excision (the argument for this was omitted in Section 4 ), we can therefore calculate $D(C(X), B)$ for any finite $C W$-complex $X$. Secondly, recall that thanks to the work of Cuntz in [4] we know that the Pimsner-Voiculescu exact sequence and Connes' Thom isomorphism hold for the functor $D(A,-)$. The contravariant version of Cuntz's results show that the same is true for $D(-, B)$. Finally, the pairings obtained in Section 3 give us immediately that $D(A, B)=D\left(A_{1}, B_{1}\right)$ when $A$ is $E$-theory equivalent to $A_{1}$ and $B$ is $E$-theory equivalent to $B_{1}$. In fact, it seems that the only major tool for the calculation of $K K$-theory and $E$-theory which at this point is not readily available for $D$ theory is the universal coefficient theorem of Rosenberg and Schochet, [13]. We may return to this point in a future publication.

\footnotetext{
${ }^{1}$ Since we are working in the category of $G$-algebras split-exactness refers to extensions of $G$-algebras which split via an equivariant $*$-homomorphism.
} 
Concerning calculations of $K J(A, B)$ we only remark that $K J(A, B)=$ $D(A, B)$ when $A$ is nuclear (and $G=0$ ).

\section{REFERENCES}

1. Brown, L. G., Continuity of actions of groups and semigroups on Banach spaces, J. London Math. Soc. 62 (2000), 107-116.

2. Connes, A., Noncommutative Geometry, Academic Press, San Diego, 1994.

3. Connes, A. and Higson, N., Deformations, morphisms asymptotiques et $K$-theorie bivariante, C.R. Acad. Sci. Paris Sér. I Math. 313 (1990), 101-106.

4. Cuntz, J., $K$-theory and $C^{*}$-algebras, Lecture Notes in Math. 1046 (1984), 55-76.

5. Dadarlat, M., A note on asymptotic morphisms, $K$-theory 8 (1994), 465-482.

6. Dadarlat, M. and Loring, T., A universal multicoefficient theorem for the Kasparov groups, Duke Math. J. 84 (1996), 355-377.

7. Dadarlat, M. and Loring, T., K-homology, asymptotic representations and unsuspended $E$ theory, J. Funct. Anal. 126 (1994), 367-383.

8. Guentner, E., Higson, N. and Trout, J., Equivariant E-theory for $C^{*}$-algebras, Mem. Amer. Math. Soc. 148 (2000).

9. Houghton-Larsen, T. and Thomsen, K., Universal (co)homology theories, K-Theory 16 (1999), 1-27.

10. Kasparov, G., Equivariant $K K$-theory and the Novikov conjecture, Invent. Math. 91 (1988), 513-572.

11. Kasparov, G., The operator $K$-functor and extensions of $C^{*}$-algebras, Izv. Akad. Nauk. SSSR, Ser. Mat. 44 (1980), 571-636.

12. Manuilov, V. M. and Thomsen, K., Quasidiagonal extensions and sequentially trivial asymptotic homomorphisms, Adv. Math. 154 (2000), 258-279.

13. Rosenberg, J. and Schochet, C., The Kunneth theorem and the universal coefficient theorem for Kasparov's generalized K-functor, Duke Math. J. 55 (1987), 431-474.

14. Rørdam, M., Classification of certain infinite simple $C^{*}$-algebras, J. Funct. Anal. 131 (1995), 415-458.

15. Samuel, J., Asymptotic morphisms and E-theory, Fields Institute Communications, 13 (1997), 291-314.

16. Thomsen, K., Equivariant KK-theory and $C^{*}$-extensions, $K$-theory 19 (2000), 219-249.

17. Thomsen, K., Asymptotic homomorphisms and equivariant KK-theory, J. Funct. Anal. 163 (1999), 324-343.

INSTITUT FOR MATEMATISKE FAG

NY MUNKEGADE

8000 AARHUS C

DENMARK

E-mail: matkt@imf.au.dk 